\title{
Wikidata through the Eyes of DBpedia
}

\author{
Ali Ismayilov ${ }^{1}$, Dimitris Kontokostas ${ }^{2}$, Sören Auer ${ }^{1}$, Jens Lehmann $^{2}$, and \\ Sebastian Hellmann ${ }^{2}$ \\ 1 University of Bonn, Enterprise Information Systems and Fraunhofer IAIS \\ s6alisma@uni-bonn.delauer@cs.uni-bonn.de \\ 2 Universität Leipzig, Institut für Informatik, AKSW \\ \{lastname\}@informatik.uni-leipzig.de
}

\begin{abstract}
DBpedia is one of the first and most prominent nodes of the Linked Open Data cloud. It provides structured data for more than 100 Wikipedia language editions as well as Wikimedia Commons, has a mature ontology and a stable and thorough Linked Data publishing lifecycle. Wikidata, on the other hand, has recently emerged as a user curated source for structured information which is included in Wikipedia. In this paper, we present how Wikidata is incorporated in the DBpedia eco-system. Enriching DBpedia with structured information from Wikidata provides added value for a number of usage scenarios. We outline those scenarios and describe the structure and conversion process of the DBpediaWikidata dataset.
\end{abstract}

Keywords: DBpedia, Wikidata, RDF

\section{Introduction}

DBpedia is one of the first and most prominent nodes of the Linked Open Data cloud. It provides structured data for more than 100 Wikipedia language editions as well as Wikimedia Commons, has a mature ontology and a stable and thorough Linked Data publishing lifecycle. Wikidata has recently emerged as a user curated source for structured information which is included in Wikipedia.

DBpedia uses human-readable Wikipedia article identifiers to create IRIs for concepts in each Wikipedia language edition and uses RDF and Named Graphs as its original data model. Wikidata on the other hand uses languageindependent numeric identifiers and developed its own data model, which provides better means for capturing provenance information. The multilingual DBpedia ontology, organizes the extracted data and integrates the different language editions while Wikidata is rather schemaless, providing only simple templates and attribute recommendations. All DBpedia data is extracted from Wikipedia and Wikipedia authors thus unconciously also curate the DBpedia knowledge base. Wikidata on the other hand has its own data curation interface, which is also based on the MediaWiki framework. DBpedia publishes a number of datasets for each language edition in a number of Linked Data ways, including datasets dumps, dereferencable resources and SPARQL endpoints. While DBpedia covers 
a very large share of Wikipedia at the expense of partially reduced quality, Wikidata covers a significantly smaller share, but due to the manual curation with higher quality and provenance information. As a result of this complementarity, aligning both efforts in a loosely coupled way would render a number of benefits for users. Wikidata would be better integrated into the network of Linked Open Datasets and Linked Data aware users had a coherent way to access Wikidata and DBpedia data. Applications and use cases have more options for choosing the right balance between coverage and quality.

In this article we describe the integration of Wikidata into the DBpedia Data Stack. People are not used to the currently very evolving Wikidata schema, while DBpedia has a relatively stable and commonly used ontology. As a result, with the DBpedia Wikidata $(\mathrm{DBw})$ dataset can be queried with the same queries that are used with DBpedia.

\section{Background}

Wikidata [5] is community-created knowledge base to manage factual information of Wikipedia and its sister projects operated by the Wikimedia Foundation. As of March 2015, Wikidata contains more than 17.4 million items and 58 million statements. The growth of Wikidata attracted researchers in Semantic Web technologies. In 2014, an RDF export of Wikidata was introduced [2] and recently a few SPARQL endpoints were made available as external contributions. Wikidata is a collection of entity pages. There are three types of entity pages: items, property and query. Every item page contains labels, short description, aliases, statements and site links. As described in the following listing (cf. 2, Figure 3]), each statement consists of a claim and an optional reference. Each claim consists of a property - value pair, and optional qualifiers. 3

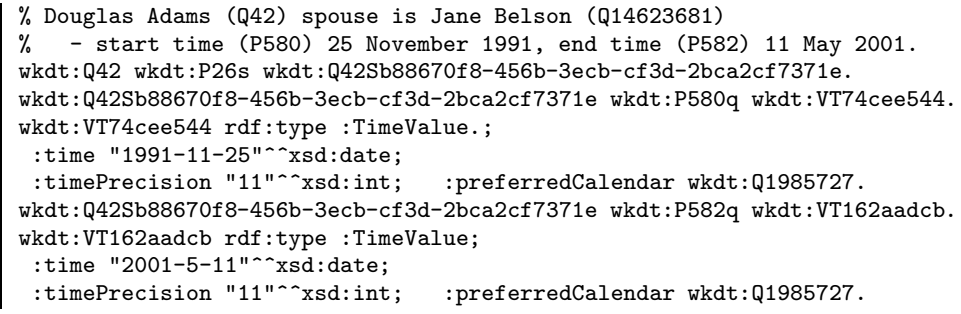

DBpedia 4] The semantic extraction of information from Wikipedia is accomplished using the DBpedia Information Extraction Framework (DIEF). The DIEF is able to process input data from several sources provided by Wikipedia. The actual extraction is performed by a set of pluggable Extractors, which rely on certain Parsers for different data types. Since 2011, DIEF is extended to provide better knowledge coverage for internationalized content 3 and allows the easier integration of different Wikipedia language editions.

\footnotetext{
3 @prefix wkdt: < http : //wikidata.org/entity/ > .
} 


\section{Conversion Process}

The DBpedia Information Extraction Framework observed major changes to accommodate the extraction of data in Wikidata. The major difference between Wikidata and the other Wikimedia projects DBpedia extracts is that Wikidata uses JSON instead of WikiText to store items.

In addition to some DBpedia provenance extractors that can be used in any MediaWiki export dump, we defined 10 additional Wikidata extractors to export as much knowledge as possible out of Wikidata. These extractors can get labels, aliases, descriptions, different types of sitelinks, references, statements and qualifiers. For statements we define a RawWikidataExtractor that extracts all available information but uses our reification scheme (cf. Section 4) and the Wikidata properties and the R2RWikidataExtractor that uses a mapping-based approach to map, in real-time, Wikidata statements to the DBpedia ontology.

Wikidata Property Mappings In the same way the DBpedia mappings wiki defines infobox to ontology mappings, in the context of this work we define Wikidata property to ontology mappings. Wikidata property mappings can be defined both as Schema Mappings and as Value Transformation Mappings.

Schema Mappings The DBpedia mappings wik is a community effort to map Wikipedia infoboxes to the DBpedia ontology and at the same time crowdsource the DBpedia ontology. Mappings between DBpedia properties and Wikidata properties are expressed as owl:equivalentProperty links in the property definition pages, e.g. dbo:birthPlace is equivalent to wkdt:P5695 Although Wikidata does not define class in terms of RDFS or OWL we use OWL punning to define owl:equivalentClass links between the DBpedia classes and the related Wikidata items, e.g. dbo:Person is equivalent to wkdt:Q5 6

Value Transformations At the time of writing, the value transformation takes the form of a JSON structure that binds a Wikidata property to one or more value transformation strings. A complete list of the existing value transformation mappings can be found in the DIEF.7 The value transformation strings that may contain special placeholders in the form of a '\$' sign as functions. If no '\$” placeholder is found, the mapping is considered constant. e.g. "P625": \{"rdf:type": "geo:SpatialThing"\}. In addition to constant mappings, one can define the following functions:

$\$ 1$ replaces the placeholder with the raw Wikidata value. e.g.

"P1566": \{"owl:sameAs": "http://sws.geonames.org/\$1/"\}.

$\$ 2$ replaces the placeholder with a space the wiki-title value, used when the value is a Wikipedia title and needs proper whitespace escaping. e.g.

"P154": \{"logo": "http://commons.wikimedia.org/wiki/Special:FilePath/\$2"\}," .

\$getDBpediaClass Using the schema class mappings, tries to map the current value to a DBpedia class. This function is used to extract rdf:type and rdfs:subClass0f statement from the respective Wikidata properties. e.g

\footnotetext{
4 http://mappings.dbpedia.org

5 http://mappings.dbpedia.org/index.php/OntologyProperty:BirthDate

6 http://mappings.dbpedia.org/index.php/OntologyClass:Person

7 https://github.com/dbpedia/extraction-framework/blob/2ab6a15d8ecd5fc9dc6ef971a71a19ad4f608ff8/du
} 
"P31": \{"rdf:type": "\$getDBpediaClass"\}

"P279": \{"rdfs:subClass0f": "\$getDBpediaClass"\}

\$getLatitude, \$getLongitude \& \$getLongitude Geo-related functions to extract coordinates from values. Following is a complete geo mapping that the extracts geo coordinates similar to the DBpedia coordinates dataset. For every occurrence of the property P625, four triples - one for every mapping are generated:
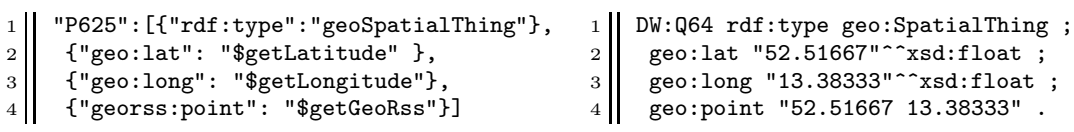

Mappings Application The R2RWikidataExtractor merges the schema \& value transformation property mappings and for every statement or qualifier it encounters, if mappings for the current Wikidata property exist, it tries to apply them and emit the mapped triples.

Additions and Post Processing Steps Besides the basic extraction phase, additional processing steps are added in the workflow.

Type Inferencing In a similar way DBpedia calculates transitive types for every resource, the DBpedia Information Extraction Framework was extended to generate these triples directly at extraction time. As soon as an rdf:type triple is detected from the mappings, we try to identify the related DBpedia class. If a DBpedia class is found, all super types are assigned to a resource.

Transitive Redirects DBpedia has already scripts in place to identify, extract and resolve redirects. After the redirects are extracted, a transitive redirect closure (excluding cycles) is calculated and applied in all generated datasets by replacing the redirected IRIs to the final ones.

Validation The DBpedia extraction framework already takes care of the correctness of the extracted datatypes during extraction. We provide two additional steps of validation. The first step is performed in real-time during extraction and checks if the property mappings has a compatible rdfs:range (literal or IRI) with the current value. The rejected triples are stored for feedback to the DBpedia mapping community. The second step is performed in a post-processing step and validates if the type of the object IRI is disjoint with the rdfs:range of the property. These errors, although they are excluded from the SPARQL endpoint and the Linked Data interface, are offered for download.

IRI Schemes As mentioned earlier, we decided to generate the RDF datasets under the wikidata.dbpedia.org domain. For example, wkdt: Q42 will be transformed to $d w: Q 428$.

Reification In contrast to Wikidata, simple RDF reification was chosen for the representation of qualifiers. This lead to a simpler design and further reuse of the DBpedia properties. The IRI schemes for the rdf:Statement IRIs follow the same verbose approach from DBpedia to make them easily writable manually

\footnotetext{
${ }^{8}$ Oprefix dw: <http://wikidata.dbpedia.org/resource/> .
} 


\begin{tabular}{|l|r|l|}
\hline Title & Triples & Description \\
\hline Provenance & $17,771,394$ & PageIDs \& revisions \\
\hline Redirects & 434,094 & Explicit \& transitive redirects \\
\hline Aliases & $4,922,617$ & Resource aliases with dbo:alias \\
\hline Labels & $61,668,295$ & Labels with rdfs:label \\
\hline Descriptions & $95,667,863$ & Descriptions with dbo:description \\
\hline Sitelinks & $41,543,058$ & DBpedia inter-language links \\
\hline Wikidata links & $17,562,043$ & Links to original Wikidata URIs \\
\hline Mapped facts & $90,882,327$ & Aggregated mapped facts \\
\hline - Types & $8,579,745$ & Direct types from the DBpedia ontology \\
\hline - Transitive Types & $48,932,447$ & Transitive types from the DBpedia ontology \\
\hline - Coordinates & $6,415,120$ & Geo coordinates \\
\hline - Images & $1,519,368$ & Depictions using foaf:depiction \& dbo:thumbnail \\
\hline - mappings & $22,270,694$ & Wikidata statements with DBpedia ontology \\
\hline - External links & $3,164,953$ & sameAs links to external databases \\
\hline Mapped facts (R) & $138,936,782$ & Mapped statements reified (all) \\
\hline Mapped facts (RQ) & 626,648 & Mapped qualifiers \\
\hline Raw facts & $59,458,835$ & Raw simple statements (not mapped) \\
\hline Raw facts (R) & $237,836,221$ & Raw statements reified \\
\hline Raw facts (RQ) & $1,161,294$ & Raw qualifiers \\
\hline References & $34,181,399$ & Reified statements references with dbo:reference \\
\hline Mapping Errors & $2,711,114$ & Facts from incorrect mappings \\
\hline Ontology Errors & 3,398 & Facts excluded due to ontology inconsistencies \\
\hline
\end{tabular}

Table 1. Description of the DBw datasets. (R) stands for a reified dataset and (Q) for a qualifiers dataset

by following a specific pattern. When the value is an IRI (Wikidata Item) then for a subject IRI Qs, a property Px and a value IRI Qv the reified statement IRI has the form dw:Qs_Px_Qv. When the value is a Literal then for a subject IRI Qs, a property Px and a Literal value Lv the reified statement IRI has the form $\mathrm{dw}$ : Qs_Px_H(Lv, 5$)$, where $H()$ is a hash function that takes as argument a string $(\mathrm{Lv})$ and a number to limit the size of the returned hash (5). The use of the hash function in the case of literals guarantees the IRI uniqueness and the value ' 5 ' is safe enough to avoid collisions and keep it short at the same time. The equivalent representation of the Wikidata example in Section 2 is: 9

1 ||dw:Q42_P26_Q14623681 a rdf:Statement ;

2 rdf:subject dw:Q42;

3 rdf:predicate dbo:spouse ;
4 rdf:object dw:Q14623681;

5 dbo: startDate "1991-11-25" ^xsd:date ;

6 dbo:endDate "2001-5-11" ^xsd:date ;

\section{Dataset Description}

A statistical overview of the DBw dataset is provided in Table 1. We extract provenance information, e.g. the MediaWiki page and revision IDs as well as redirects. Aliases labels and descriptions are extracted from the related Wikidata item section and are similar to the RDF data Wikidata provides. A difference to Wikidata are the properties we chose to associate aliases and description.

Wikidata sitelinks are processed to provide three datasets: 1) owl:sameAs links between DBw IRIs and Wikidata IRIs (e.g. dw:Q42 owl:sameAs wkdt:Q42), 2) owl:sameAs links between DBW IRIs and sitelinks converted to DBpedia IRIs (e.g. dw:Q42 owl:sameAs db-en:Douglas_Adams) and 3) for every language in the mappings wiki we generate owl:sameAs links to all other languages (e.g.

\footnotetext{
${ }^{9} \mathrm{DBw}$ does not provide precision. Property definitions exist in the DBpedia ontology
} 


\begin{tabular}{|l|r|}
\hline Class & Count \\
\hline dbo:Agent & $2,884,505$ \\
\hline dbo:Person & $2,777,306$ \\
\hline geo:spatialThing & $2,153,258$ \\
\hline dbo:TopicalConcept & $1,907,203$ \\
\hline dbo:Taxon & $1,906,747$ \\
\hline
\end{tabular}

Table 2. Top classes

\begin{tabular}{|l|r|}
\hline Property & Count \\
\hline dbo:date & 301,085 \\
\hline dbo:startDate & 158,947 \\
\hline geo:point & 108,526 \\
\hline dbo:endDate & 50,058 \\
\hline dbo:country & 33,698 \\
\hline
\end{tabular}

Table 4. Top mapped qualifiers

\begin{tabular}{|l|r|}
\hline Property & Count \\
\hline Owl:sameAs & $232,890,848$ \\
\hline rdf:type & $145,407,453$ \\
\hline dbo:description & $95,667,863$ \\
\hline rdfs:label & $61,704,172$ \\
\hline rdfs:seeAlso & $5,125,945$ \\
\hline
\end{tabular}

Table 3. Top properties

\begin{tabular}{|l|r|}
\hline Property & Count \\
\hline wd:P31 & $13,070,656$ \\
\hline wd:P17 & $3,051,166$ \\
\hline wd:P21 & $2,604,741$ \\
\hline wd:P131 & $2,372,237$ \\
\hline wd:P625 & $2,167,100$ \\
\hline
\end{tabular}

Table 5. Top properties in Wikidata

$\mathrm{db}-\mathrm{en}$ :Douglas_Adams owl:sameAs db-de:Douglas_Adams). The latter is used for the DBpedia releases in order to provide links between the different DBpedia language editions.

Mapped facts are generated from the Wikidata property mappings (cf. Section 3]). Based on a combination of the predicate and object value of a triple they are split in different datasets. Types, transitive types, geo coordinates, depictions and external owl:sameAs links are separated. The rest of the mapped facts are in the mappings dataset. The reified mapped facts $(\mathrm{R})$ contains all the mapped facts as reified statements and the mapped qualifiers for these statements (RQ) are provided separate (cf. Listing 3).

Raw facts consist of three datasets that generate triples with DBw IRIs and the original Wikidata properties. The first dataset (raw facts) provides triples for simple statements. The same statements are reified in the second dataset $(\mathrm{R})$ and in the third dataset (RQ) we provide qualifiers linked in the reified statements. Example of the raw datasets can be seen in Listing 3 by replacing the DBpedia properties with the original Wikidata properties. These datasets provide full coverage and, except from the reification design and different namespace, can be seen as equivalent with the WikidataRDF dumps.

Wikidata statement references are extracted in the references dataset using the reified statement resource IRI as subject and the dbo:reference property. Finally, in the mapping and ontology errors datasets we provide triples rejected according to Section 3.

\section{DBpedia WikiData In Use}

Statistics and Evaluation The statistics we present are based on the Wikidata XML dump from March 2015. We managed to generate a total of 1B triples with 131,926,822 unique resources. In Table 1 we provide the number of triples per combined datasets.

Class 8 property statistics We provide the 5 most popular DBW classes in Table 2. We managed to extract a total of 6.5M typed Things with Agents and SpatialThing as the most frequent types. The 5 most frequent mapped properties in simple statements are provided in Table 3 and the most popular mapped properties in qualifiers in Table 4. Wikidata does not have a complete range of value types and date paoperties are the most frefuent at the moment. 
Mapping statistics In total, 270 value transformation mappings were defined along with 163 owl:equivalentProperty and 318 owl:equivalentClass schema mappings. Wikidata has 1465 properties defined with a total of $60,119,911$ occurrences. With the existing mappings we covered $77.2 \%$ of the occurrences.

Redirects In the current dataset we generated 434,094 redirects - including transitive. When these redirects were applied to the extracted datasets, they replaced 1,161,291 triples to the redirected destination. The number of redirects in Wikidata is small compared to the project size but is is also a relatively new project. As the project matures in time the number of redirects will increase and resolving them will have an impact on the resulting data.

Validation According to Table 1, a total of 2.7M errors originated from schema mappings and 3,398 triples did not pass the ontology validation (cf. Section 3).

Access and Sustainability This dataset will be part of the official DBpedia knowledge infrastructure and be published through the regular releases of DBpedia, along with the rest of the DBpedia language editions. The first DBpedia release that will include this dataset is due on April - May 2015 (2015A). DBpedia is a pioneer in adopting and creating best practices for Linked Data and RDF publishing. Thus, being incorporated into the DBpedia publishing workflow guarantees: a) long-term availability through the DBpedia Association and b) agility in following best-practices as part of the DBpedia Information Extraction Framework. In addition to the regular and stable releases of DBpedia we provide more frequent dataset updates from the project website 10

Besides the stable dump availability we created http://wikidata.dbpedia.org for the provision of a Linked Data interface and a SPARQL Endpoint. The dataset is registered in DataHul 11 and provides machine readable metadata as void 12 and DataID 13 1. Since the project is now part of the official DBpedia Information Extraction Framework, our dataset reuses the existing user and developer support infrastructure. DBpedia has a general discussion and developer list as well as an issue tracker 14 for submitting bugs.

Use Cases Although it is early to identify all possible use cases for DBw, our main motivation was a) ease of use, b) vertical integration with the existing DBpedia infrastructure and c) data integration and fusion. Following we list SPARQL query examples for simple and reified statements. Since DBpedia provides transitive types directly, queries where e.g. someone asks for all 'places' in Germany can be formulated easier. Moreover, dbo: country can be more intuitive than wkdt:P17c. Finally, the DBpedia queries can, in most cases directly or with minor adjustments, run on all DBpedia language endpoints. When someone is working with reified statents, the DBpedia IRIs encode all possible information to visually identify the resources and items involved (cf. Section 3) in the

10 http://wikidata.dbpedia.org/downloads

11 http://datahub.io/dataset/dbpedia-wikidata

12 http://wikidata.dbpedia.org/downloads/void.ttl

13 http://wikidata.dbpedia.org/downloads/20150330/dataid.ttl

14 https://github.com/dbpedia/extraction-framework/issues 
statement while Wikidata uses a hash string. In addition, querying for reified statement in Wikidata needs to properly suffix the Wikidata property with c/s/q:

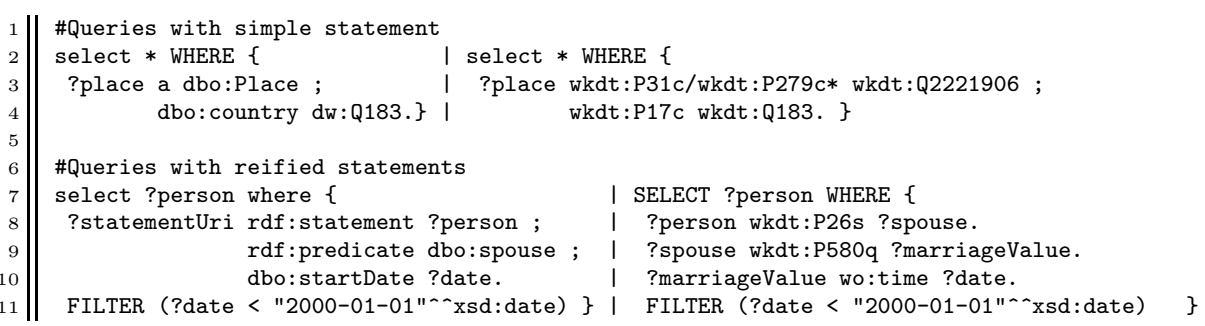

An additional important use case is data integration. Converting a dataset to a more used and well-known schema, it makes it easier to integrate the data. The fact the datasets are split according to the information they contain makes data consumption easier when someone needs a specific subset, e.g. coordinates. The DBw dataset is also planned to be used as an enrichment dataset on top of DBpedia and fill in semi-structured data that are being moved to Wikidata. It is also part of short-term plan to fuse all DBpedia data into a single namespace and the DBw dataset will have a prominent role in this effort.

\section{Conclusions and Future Work}

We present an effort to provide an alternative RDF representation of Wikidata. Our work involved the creation of 10 new DBpedia extractors, a Wikidata2DBpedia mapping language and additional post-processing \& validation steps. With the current mapping status we managed to generate over 1 billion RDF triples. In the future we plan to extend the mapping coverage as well as extend the language with new mapping functions and more advanced mapping definitions.

\section{References}

1. M. Brümmer, C. Baron, I. Ermilov, M. Freudenberg, D. Kontokostas, and S. Hellmann. DataID: Towards semantically rich metadata for complex datasets. In Proc. of the 10th International Conference on Semantic Systems, pages 84-91. ACM, 2014.

2. F. Erxleben, M. Günther, M. Krötzsch, J. Mendez, and D. Vrandečić. Introducing Wikidata to the linked data web. In ISWC'14, LNCS. Springer, 2014.

3. D. Kontokostas, C. Bratsas, S. Auer, S. Hellmann, I. Antoniou, and G. Metakides. Internationalization of linked data: The case of the greek dbpedia edition. Web Semantics:Science,Services $\&$ Agents on the World Wide Web, 15(0):51 - 61, 2012.

4. J. Lehmann, R. Isele, M. Jakob, A. Jentzsch, D. Kontokostas, P. N. Mendes, S. Hellmann, M. Morsey, P. van Kleef, S. Auer, and C. Bizer. DBpedia - a large-scale, multilingual knowledge base extracted from wikipedia. SWJ, 6(2):167-195, 2015.

5. D. Vrandečić and M. Krötzsch. Wikidata: A free collaborative knowledgebase. Commun. ACM, 57(10):78-85, Sept. 2014. 